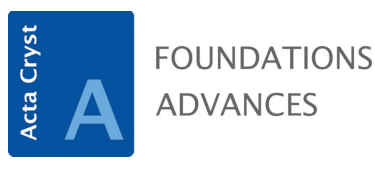

ISSN 2053-2733

Received 7 September 2018

Accepted 10 December 2018

Edited by A. I. Goldman, lowa State University, USA

Keywords: intermetallic phases; channel structures; chemical bonding theory; disorder; incommensurability.

CCDC references: $1880447 ; 1880448$; 1880449; 1880452

Supporting information: this article has supporting information at journals.iucr.org/a

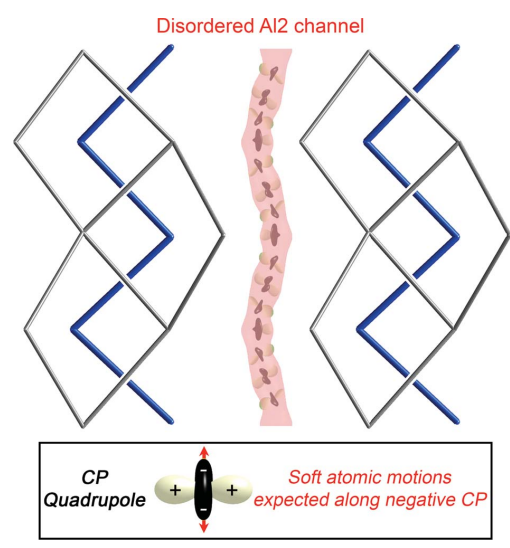

OPEN $\odot$ ACCESS

\section{Principles of weakly ordered domains in intermetallics: the cooperative effects of atomic packing and electronics in $\mathrm{Fe}_{2} \mathrm{Al}_{5}$}

\author{
Anastasiya I. Vinokur, Katerina P. Hilleke and Daniel C. Fredrickson* \\ Department of Chemistry, University of Wisconsin-Madison, 1101 University Avenue, Madison, WI 53706, USA. \\ *Correspondence e-mail: danny@chem.wisc.edu
}

Many complex intermetallic structures feature a curious juxtaposition of domains with strict 3D periodicity and regions of much weaker order or incommensurability. This article explores the basic principles leading to such arrangements through an investigation of the weakly ordered channels of $\mathrm{Fe}_{2} \mathrm{Al}_{5}$. It starts by experimentally confirming the earlier crystallographic model of the high-temperature form, in which nearly continuous columns of electron density corresponding to disordered $\mathrm{Al}$ atoms emerge. Then electronic structure calculations on ordered models are used to determine the factors leading to the formation of these columns. These calculations reveal electronic pseudogaps near 16 electrons $/ \mathrm{Fe}$ atom, an electron concentration close to the Al-rich side of the phase's homogeneity range. Through a reversed approximation Molecular Orbital (raMO) analysis, these pseudogaps are correlated with the filling of 18electron configurations on the $\mathrm{Fe}$ atoms with the support of isolobal $\sigma \mathrm{Fe}-\mathrm{Fe}$ bonds. The resulting preference for 16 electrons/Fe requires a fractional number of $\mathrm{Al}$ atoms in the $\mathrm{Fe}_{2} \mathrm{Al}_{5}$ unit cell. Density functional theory-chemical pressure (DFT-CP) analysis is then applied to investigate how this nonstoichiometry is accommodated. The $\mathrm{CP}$ schemes reveal strong quadrupolar distributions on the $\mathrm{Al}$ atoms of the channels, suggestive of soft atomic motions along the undulating electron density observed in the Fourier map that allow the Al positions to shift easily in response to compositional changes. Such a combination of preferred electron counts tied to stoichiometry and continuous paths of $\mathrm{CP}$ quadrupoles could provide predictive indicators for the emergence of channels of disordered or incommensurately spaced atoms in intermetallic structures.

\section{Introduction}

The structural chemistry of intermetallic phases is rich in phenomena that go beyond simple notions of translational symmetry in 3D space, such as disorder, incommensurability and quasicrystalline arrangements. In each of these scenarios, weakly specified atomic positions are often distributed within frameworks of rigidly positioned atoms with a variety of morphologies. In some cases, such as the disordered tetrahedra at the centers of the icosahedral building units of Tsaitype quasicrystals and their approximants (Gómez \& Lidin, 2003; Piao et al., 2006; Takakura et al., 2007), the less organized regions can be isolated to small pockets of the structure. Disordered or incommensurately spaced atoms can also run along features of higher dimensionality: 1D columns (Mählpfordt, 1997; Rohrer et al., 2001; Piao \& Lidin, 2007; Kanno et al., 2017) or 2D planes (Latturner \& Kanatzidis, 2002; Gray et al., 2008; Kilduff \& Fredrickson, 2016). In the most extreme examples, as in the classic $\beta-\mathrm{Mg}_{2} \mathrm{Al}_{3}$ structure (Samson, 1965; Feuerbacher et al., 2007), the scrambled atoms can form 3D continuous sublattices that permeate the whole 
crystal. Such a duality of strict and loose order within the same structure offers immense opportunities for material properties, e.g. for the realization of the phonon-glass electron crystal concept (Nolas et al., 1999), or the creation of well defined paths for atomic diffusion (Mehrer, 1996). However, the absence of periodic order in these situations makes their design from first-principles calculations challenging. In this article, we will work towards deriving chemical principles for the emergence of such features in intermetallics, using the channels of weakly ordered atoms in the $\mathrm{Fe}_{2} \mathrm{Al}_{5}$ structure as a model system.

As with many other non-trivial intermetallic compounds, the structure of $\mathrm{Fe}_{2} \mathrm{Al}_{5}$ (a phase whose homogeneity range extends from approximately $\mathrm{Fe}_{2} \mathrm{Al}_{4.67}$ to $\mathrm{Fe}_{2} \mathrm{Al}_{5.4}$ ) has gradually emerged over the course of decades in successive investigations. After an initial report of an $\mathrm{FeAl}_{2}$ phase in 1933 (Osawa, 1933), the stoichiometry of the phase was revised in 1953 to $\mathrm{Fe}_{2} \mathrm{Al}_{5}$, and the compound was described in passing as being structurally similar to the hexagonal phase $\mathrm{Co}_{2} \mathrm{Al}_{5}$ (Schubert et al., 1953). Subsequent investigations revealed that, on the contrary, it adopts its own structure type with little relation to that of $\mathrm{Co}_{2} \mathrm{Al}_{5}$ (Van der Kraan \& Buschow, 1986; Ellner \& Mayer, 1992). In 1994, a crystal structure determination revealed nearly continuous columns of electron density passing through the structure (Fig. 1), which were modeled as chains of tightly spaced, fractionally occupied $\mathrm{Al}$ atoms (Burkhardt et al., 1994). This extensive positional disorder inspired numerous investigations into the phase's properties and stability. $\mathrm{Fe}_{2} \mathrm{Al}_{5}$ was discovered to behave as a dilute magnet (Chi et al., 2010; Jagličić et al., 2011), while phonon band structure calculations revealed fluidity along the disordered channels (Mihalkovič \& Widom, 2012) in accord with the heat capacity measurements of Chi et al. (2010). Such soft motions are suggestive of low thermal conductivity, highlighting potential for thermoelectric applications that was recently realized experimentally (Tobita et al., 2016). Finally, in the latest structural studies, lowtemperature polymorphs exhibiting superstructure formation or incommensurate order were characterized with selectedarea electron diffraction and powder X-ray diffraction (Becker et al., 2017; Okamoto et al., 2017; Becker \& Leineweber, 2018).

As the behavior of the $\mathrm{Al}$ atoms in this column and the consequent potential for physical properties have emerged with greater clarity, a number of questions of structural chemistry remain unresolved. What drives the formation of these weakly ordered channels? How could similar situations be staged in other intermetallic compounds? Motivated by these questions, we will investigate the electronic structure of $\mathrm{Fe}_{2} \mathrm{Al}_{5}$, correlating its structural features to the $18-n$ bonding scheme (Yannello \& Fredrickson, 2014, 2015) and to chemical pressure (CP) distributions (Fredrickson, 2012;
Berns et al., 2014). Using the reversed approximation Molecular Orbital (raMO) analysis (Yannello et al., 2014), the Al nonstoichiometry will be linked to the preferred electron count of the $\mathrm{Fe}$ bonding network. The localization of the nonstoichiometry to the weakly ordered channels will then be connected to the presence of $\mathrm{CP}$ quadrupoles on the corresponding $\mathrm{Al}$ atoms, a feature associated with soft atomic motions (Engelkemier \& Fredrickson, 2016). $\mathrm{Fe}_{2} \mathrm{Al}_{5}$ will thus exemplify complex structures in which disordered regions arise from cooperation between electronic and atomic packing effects, where a feature driven by electronic effects is guided by $\mathrm{CP}$, a theme we anticipate to play a broad role in intermetallic chemistry.

\section{Technical procedures}

\subsection{Experimental procedures}

To confirm its structural features, the high-temperature form of $\mathrm{Fe}_{2} \mathrm{Al}_{5}$ was synthesized by first grinding together powders of the pure elements (Fe powder, $99.9 \%$, Strem; $\mathrm{Al}$ powder $-100+325$ mesh, $99.97 \%$, Alfa Aesar) in a ratio of 1:2.7 with an agate mortar and pestle in an Ar-filled glovebox. The mixtures were then pressed into pellets and arc-melted under Ar two times for $10 \mathrm{~s}$ each (to maximize homogeneity while minimizing $\mathrm{Al}$ loss by evaporation). The pellets were sealed in evacuated fused silica ampoules and annealed first at $873 \mathrm{~K}$ for $4 \mathrm{~d}$, then at $673 \mathrm{~K}$ for $6 \mathrm{~d}$, and finally quenched in ice water. All syntheses resulted in gray pellets with a metallic sheen that showed no visible signs of air sensitivity even after weeks in air. As described in the supporting information, the samples were characterized with single-crystal and powder X-ray diffraction, energy-dispersive X-ray spectroscopy (EDS), and wavelength-dispersive $\mathrm{X}$-ray spectroscopy (WDS).

\subsection{Ordered structure models}

For the theoretical analysis of the driving forces shaping the disordered regions within $\mathrm{Fe}_{2} \mathrm{Al}_{5}$, several ordering patterns 
were examined involving different placements of $\mathrm{Al}$ atoms over the Al2a, Al2b and Al2c sites within the two channels in the conventional cell. Five models in total were considered (designated as Models 1-5) following the occupation patterns given in Table S14 in the supporting information. For example, Model 1 was built from a $1 \times 1 \times 2$ supercell, with only every fifth atom along the $\mathrm{Al} 2 \mathrm{Al}$ columns being kept, then deleting one additional $\mathrm{Al}$ atom from one of the columns, generating the appropriate $\mathrm{Fe}_{2} \mathrm{Al}_{5.25}$ stoichiometry (15.875 electrons/Fe) and a space-group symmetry of $P m 11$ (with the non-standard setting being used to maintain the axis labels of the original cell). For Model 2, $\mathrm{Al}$ atoms were placed at every other Al2a site (space group $C 2 / m 11$, also set in a $1 \times 1 \times 2$ supercell in the calculations preliminary to the raMO analysis, for consistency), yielding a stoichiometry of $\mathrm{Fe}_{2} \mathrm{Al}_{5}$ (15.5 electrons/Fe). In Model 3, meanwhile, every other Al2b site is occupied by an $\mathrm{Al}$ atom (stoichiometry: $\mathrm{Fe}_{2} \mathrm{Al}_{5}$, space group: $\mathrm{Cm} 2 \mathrm{~m}$ ). Models 4 and 5 are variations on Models 1 and 2, respectively, in which the contents of the two weakly ordered columns in the conventional cell are shifted relative to each other, with the symmetries Pm11 and Pmnn (though, curiously, the geometrical optimization of Model 4 converged on a geometry nearly indistinguishable from Model 1). Models 1 and 2 were used for the raMO analysis, with the similar electronic density of states (DOS) features of the remaining models (Fig. S7 in the supporting information) suggesting that analogous bonding schemes apply. Density functional theory-chemical pressure (DFT-CP) analysis was carried out on all of the unique structures (Models 1-3 and 5). The stoichiometries for all models lie within the experimental phase width of the phase, where the electron concentration ranges from 15.0 to 16.1 electrons/Fe (Li et al., 2016).

\subsection{Reversed approximation Molecular Orbital analysis}

The raMO analysis was employed to analyze the role of electron count in the stability of $\mathrm{Fe}_{2} \mathrm{Al}_{5}$. The DFT electronic structures, under the generalized gradient approximation (GGA), of Models 1 and 2 were calculated with the Vienna $A b$ Initio Simulation Package (VASP) (Kresse \& Furthmüller, $1996 a, b)$ in the high-precision mode, using the projector augmented wave (PAW) potentials provided with the program (Blöchl, 1994; Kresse \& Joubert, 1999), $3 \times 3 \times 3$ Г-centered $k$-point grids and an energy cutoff of $334.9 \mathrm{eV}$. Both structures were geometrically optimized with a two-step procedure, with the atomic coordinates first being relaxed within a fixed unit cell, followed by the release of all structural parameters. Single-point calculations were then performed to obtain band energies and DOS distributions.

The resulting GGA-DFT output was then used to refine parameters for simple Hückel models with the program eHtuner (Stacey \& Fredrickson, 2012). From the finalized Hückel parameters, Hamiltonian matrices were calculated with YAeHMOP (Landrum, G. A. \& Glassey, W. V.; YAeHMOP: Yet Another extended Hückel Molecular Orbital Package, freely available at http://yaehmop.sourceforge.net/) for the $\Gamma$ points of $3 \times 3 \times 3$ supercells, which were directly input into our in-house MATLAB programs Figuretool2 and makeraMO for the raMO analyses (Yannello et al., 2014). Further details, including atomic coordinates for the models, tables of DFT-calibrated Hückel parameters and comparisons of the GGA-DFT and Hückel DOS curves, are provided in the supporting information.

\subsection{DFT-CP analysis}

DFT-CP analyses were performed on Models $1-3$ and 5 . Prior to the DFT-CP calculations, geometry optimizations were performed with DFT under the local density approximation (LDA) with the ABINIT program (Gonze et al., 2002, 2009) using Hartwigsen-Goedecker-Hutter norm-conserving pseudopotentials (Hartwigsen et al., 1998) for Models 2, 3 and 5. Because of its larger cell size, the geometry optimizations for Model 1 were performed using LDA-DFT in VASP with ultrasoft pseudopotentials (Vanderbilt, 1990). Single-point calculations were then carried out in ABINIT on these optimized geometries at the equilibrium volume as well as at slightly expanded and contracted volumes, yielding the kinetic energy densities, electron densities and local components of the Kohn-Sham potentials used in the CP analyses. Further details, including energy cutoffs and $k$-point grids, may be found in the supporting information.

$\mathrm{CP}$ maps were produced from the $A B I N I T$ output using the CPmap module of CPpackage2, including core unwarping and mapping of the nonlocal pseudopotential energies (Berns $e t$ al., 2014; Hilleke \& Fredrickson, 2018). These maps were divided among contact volumes between pairs of atoms using the binary Hirshfeld-inspired scheme in the CPintegrate module (Berns et al., 2014), averaging the CP values within the contact volumes to obtain interatomic pressures. These pressures were next projected onto real spherical harmonics $(l \leq 4)$ centered on the atomic positions, and the resulting $\mathrm{CP}$ schemes were visualized using Figuretool2. The free ion electron densities used in the core unwarping and contact volume construction procedures were created with the Atomic Pseudopotentials Engine (APE) (Oliveira \& Nogueira, 2008), using fractions of the charges obtained from a Bader charge analysis of the PAW-GGA electronic structures with the Bader program (Bader, 1990; Henkelman et al., 2006; Tang et al., 2009) or using the Hirshfeld charges (Hirshfeld, 1977) output by CPpackage2.

\subsection{Phonon band structure calculations}

The phonon band structure of Model 2 (with every other Al2a site occupied) used for the CP analysis was calculated with the ABINIT program using the linear response method (Giannozzi et al., 1991). A series of calculations were carried out, beginning with the production of a wavefunction file using a $\Gamma$-centered $k$-point grid. Subsequent non-self-consistent calculations were performed at all $q$-points corresponding to the $k$-points used in the reference calculation to obtain linear responses for displacements of individual atoms along each Cartesian axis. The interatomic force constants were determined from these linear responses using the ABINIT 
utilities $m r g d d b$ and anaddb, while the resulting phonon modes, band structures and DOS curves were plotted with Figuretool2.

\section{Results and discussion}

\subsection{Structural analysis of the high-temperature $\mathrm{Fe}_{2} \mathrm{Al}_{5}$ phase}

With an interest in the weakly ordered channels of $\mathrm{Fe}_{2} \mathrm{Al}_{5}$, we synthesized samples of its high-temperature form for structural analysis. Single crystals were picked from the crushed reaction mixture for X-ray diffraction experiments. The diffraction data collected at room temperature were indexed with an orthorhombic unit cell with $a=7.5660$ (3), $b=6.4117$ (2) and $c=4.22350$ (18) $\AA$, in close agreement with the previously reported cell (Burkhardt et al., 1994). No superstructure or satellite reflections were observed, all of the observed systematic absences were consistent with the $\mathrm{Cmcm}$ space group assigned earlier, and the structure solution shows features in accord with those Burkhardt et al. chronicled.

A simple way to visualize this structure that will be helpful for our theoretical analysis is through its relationship to the CsCl-type structure of FeAl (Fig. 2a). To derive the $\mathrm{Fe}_{2} \mathrm{Al}_{5}$ structure, we first delete half of the $\mathrm{Fe}$ atoms in $\mathrm{FeAl}$ (Fig. 2b) to leave behind $\mathrm{Fe}$ zigzag chains, creating a $\mathrm{Fe}_{2} \mathrm{Al}_{4}$ framework. We next open the framework around the new void spaces (Fig. 2c) to form octagonal channels. Finally, the structure is completed by adding $\mathrm{Al}$ atoms in a disordered chain into the channel, yielding a composition of $\mathrm{Fe}_{2} \mathrm{Al}_{4+\delta}[\delta \simeq 0.67$ to 1.4 (Fig. 2d)]. In effect, the structure is derived from $\mathrm{FeAl}$ by replacing half the $\mathrm{Fe}$ atoms with continuous columns of possible $\mathrm{Al}$ positions.

The Fourier map for the $\mathrm{Al}$ atoms in this channel shows an uninterrupted, sinusoidal column of electron density (Fig. 1). Modeling this region atomistically is somewhat arbitrary given the absence of features corresponding to discrete positions. We found it convenient to model the domain with the three partially occupied $\mathrm{Al}$ positions shown in Fig. 1(b), where two of the sites (Al2a and Al2b) correspond to maxima in the density and the third (A12c) serves to bridge them. Curiously, the $\mathrm{Al}$ content of the column consistently refined to a formula near $\mathrm{Fe}_{2} \mathrm{Al}_{5.5}$ for various models, compared with the $\mathrm{Fe}_{2} \mathrm{Al}_{5.24}$ determined from our WDS measurements. Attempts to constrain the $\mathrm{Al}$ occupancies to match the WDS result, however, led some of the $\mathrm{Al}$ atomic displacement parameters to become nonpositive definite. Such overestimation of $\mathrm{Al}$ content in the crystallographic model was also reported by Burkhardt et al., which they attributed to an incomplete absorption correction or some Fe mixing on the disordered $\mathrm{Al}$ sites. The presence of a small amount of $\mathrm{Fe}$ in this disordered region would easily explain the discrepancy between the WDS and single-crystal compositions, and was included in the models of Becker \& Leineweber (2018). However, for our present purposes, we will focus on the role $\mathrm{Al}$ atoms play in these channels, neglecting the potential presence of a minor number of $\mathrm{Fe}$ atoms.
While neither superstructure nor satellite reflections were observed at room temperature, we were curious whether ordering transitions might be observable under different conditions. We thus carried out single-crystal X-ray diffraction experiments at 100, 150 and $400 \mathrm{~K}$. In each case, no additional reflections appeared (see the supporting information), and solving the crystal structures at the three temperatures showed the same continuous column of electron density, which was still well modeled with a triad of partially occupied Al positions (Fig. 3), and showed only minimal shifts in the atom positions. For the relatively Al-rich composition of the crystal, it thus appears that the kinetics for transforming to the ordered polymorphs of $\mathrm{Fe}_{2} \mathrm{Al}_{5}$ are slow at these temperatures relative to the time-scale of the single-crystal data collection. Together, these observations highlight the weakness of the driving forces for positional order along the structure's channels.

(a) CsCl-type FeAl structure

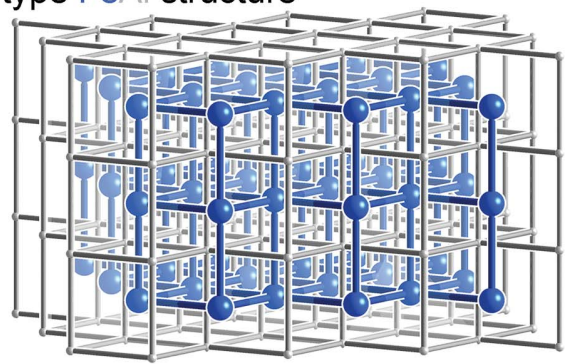

(b) Delete $1 / 2$ of the Fe atoms

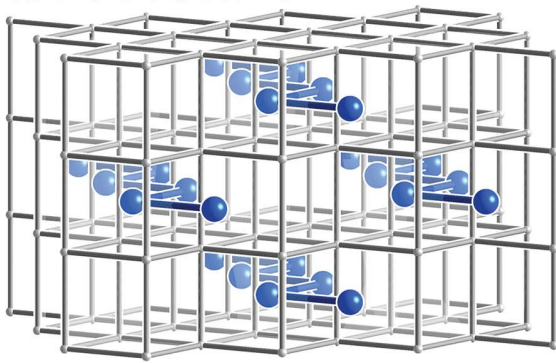

(c) Open $\mathrm{Fe}_{2} \mathrm{Al}_{4}$ framework
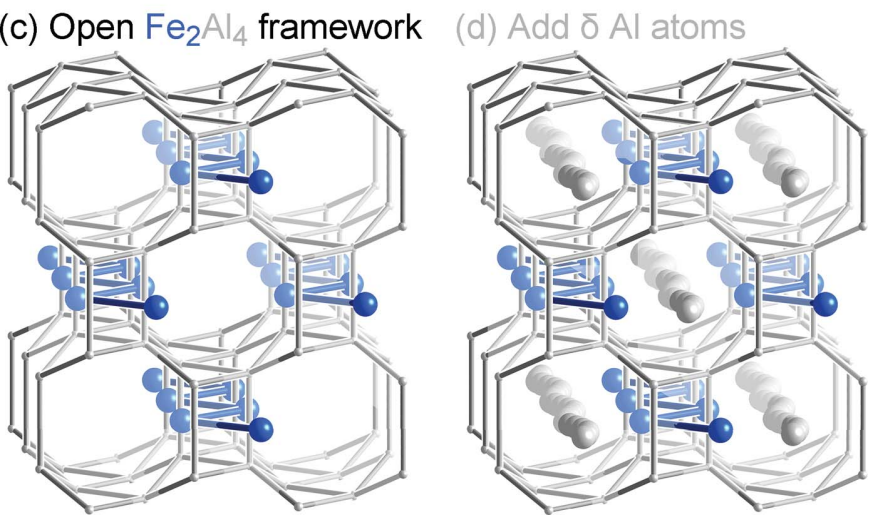

Figure 2

Derivation of the $\mathrm{Fe}_{2} \mathrm{Al}_{5}$ structure. (a) The CsCl-type structure of FeAl. (b) Deletion of half of the $\mathrm{Fe}$ atoms of $\mathrm{FeAl}$ such that the remaining ones form zigzag chains. (c) Opening the resulting $\mathrm{Fe}_{2} \mathrm{Al}_{4}$ framework creates channels ready for the inclusion of additional atoms. $(d)$ The addition of $\delta$ $\mathrm{Al}$ atoms into the channels yields a structure with the composition $\mathrm{Fe}_{2} \mathrm{Al}_{4+\delta}$. 

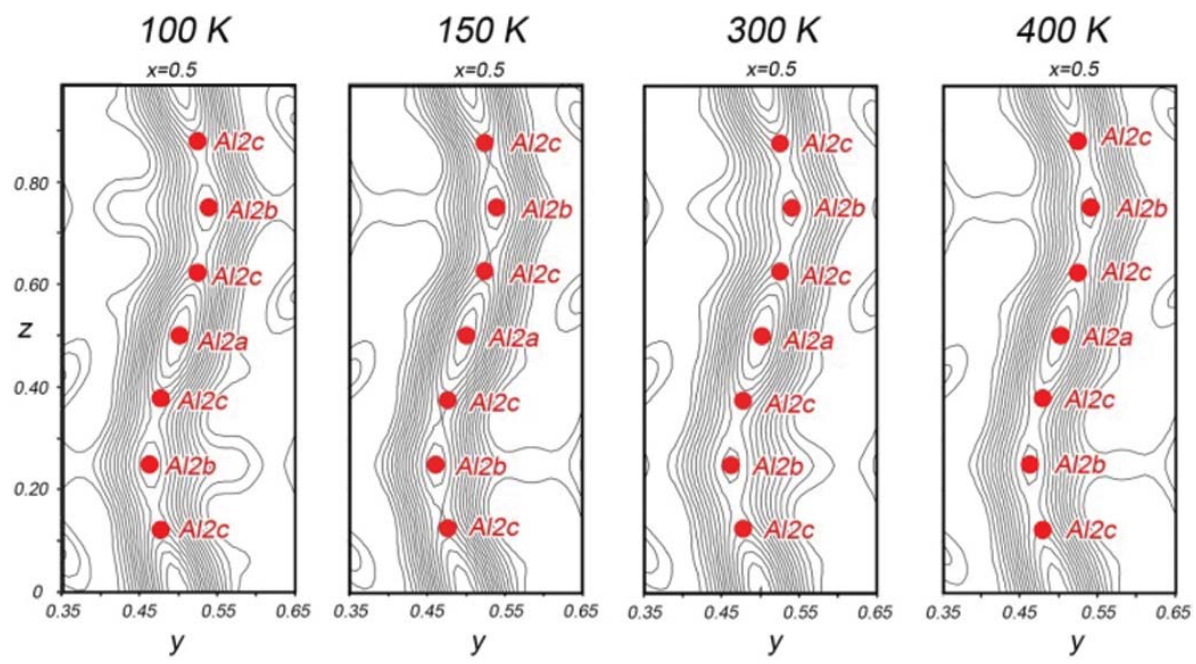

Figure 3

Evolution of the X-ray diffraction pattern and Al2a, Al2b and Al2c channel electron density as a function of temperature. The cross sections of the Fourier electron density through the Al2a, Al2b and Al2c channels obtained for data sets collected at 100, 150, 300 and $400 \mathrm{~K}$.

\subsection{Electron counting and Al nonstoichiometry}

In our structural analysis of the last section, as in previous studies, the most striking characteristic of $\mathrm{Fe}_{2} \mathrm{Al}_{5}$ is its channels of weakly ordered $\mathrm{Al}$ atoms. We now turn to exploring the role that these features play in the stability of $\mathrm{Fe}_{2} \mathrm{Al}_{5}$. The composition of this compound places it within the domain of a broad electron counting rule for transition metal-main group (T-E) intermetallic phases, the $18-n$ rule (Yannello \& Fredrickson, 2014, 2015), which can be considered as a limiting case of the more general $5 t+4 c-b$ scheme of Kitahara et al. (2015, 2017). In these compounds, the T atoms strive for filled 18-electron configurations, in which an electron pair is associated with each of their valence $s, p$ and $d$ atomic orbitals. To achieve these closed shells, each $\mathrm{T}$ atom requires $18-n$ electrons, where $n$ is the number of electron pairs the T atom shares covalently with its T-atom neighbors through multicenter E-bridged functions we refer to as isolobal T-T bonds (Yannello \& Fredrickson, 2014, 2015). Adherence to this rule is correlated with the presence of a bandgap or pseudogap in the DOS distributions at the Fermi energy $\left(E_{\mathrm{F}}\right)$, common indicators of electronic stability.

One advantage of the $18-n$ rule is that it can be simply applied to compounds where disorder occurs in the main group sublattice, as only the total electron concentration of the phase and the $\mathrm{T}-\mathrm{T}$ connectivity must be definitively known. According to the $\mathrm{Fe}-\mathrm{Al}$ phase diagram ( $\mathrm{Li}$ et al., 2016), for temperatures below circa $1273 \mathrm{~K}$, the $\mathrm{Fe}_{2} \mathrm{Al}_{5}$ homogeneity range stretches from 70.0 to 72.9 at. $\% \mathrm{Al}$, leading to a range of valence electron counts of 15.0 to 16.1 electrons/Fe atom. As the upper end of this range is approximately two electrons/Fe atom short of 18 , we expect the $\mathrm{Fe}$ atoms should each take part in two $\mathrm{Fe}-\mathrm{Fe}$ isolobal bonds, consistent with their condensation into zigzag chains in $\mathrm{Fe}_{2} \mathrm{Al}_{5}$.

Testing this picture with theoretical calculations, however, requires periodic, ordered models of the structure. As described in Section 2.2, we constructed two such GGA- electron-like distribution rich in $\mathrm{Al} s p$ character.

DFT-optimized models for detailed bonding analysis with stoichiometries of $\mathrm{Fe}_{2} \mathrm{Al}_{5.25}$ (Model 1) and $\mathrm{Fe}_{2} \mathrm{Al}_{5}$ (Model $2)$. The resulting electronic DOS distributions (Fig. 4) show strong similarities despite the differences in the placement and loading of the A12 atoms within the channels. For each model, the $E_{\mathrm{F}}$ falls close to a narrow pseudogap centered near $-8 \mathrm{eV}$ (hinting at nearly optimized bonding), lying right in the center of the DOS minimum at 15.86 electrons $/ \mathrm{Fe}$ $\left(\mathrm{Fe}_{2} \mathrm{Al}_{5.25}\right)$, and dropping to the lower shoulder at 15.5 electrons/Fe $\left(\mathrm{Fe}_{2} \mathrm{Al}_{5}\right)$. The states bracketing the pseudogap in each case are largely derived from the $\mathrm{Fe} d$ orbitals (whose contributions are shaded in black), while the lowest energy levels (between -12 and $-19 \mathrm{eV}$ ) are dominated by a nearly free

The presence of a pseudogap near 16 electrons/Fe atom is highly suggestive of the compound following the $18-n$ scheme. To make this connection more direct, we turn to the raMO analysis (Yannello et al., 2014). In the raMO approach, the occupied crystal orbitals of a system serve as a basis set for the reconstruction of localized target functions which are hypothesized to play a key role in the local bonding of the compound, for an 18-electron configuration usually the $\mathrm{T}$ atom's nine $s, p$ and $d$ valence orbitals. The raMO analysis then creates Wannier-like functions that represent the best possible orthogonal approximations to these target functions from the occupied orbitals, with the deviations from the

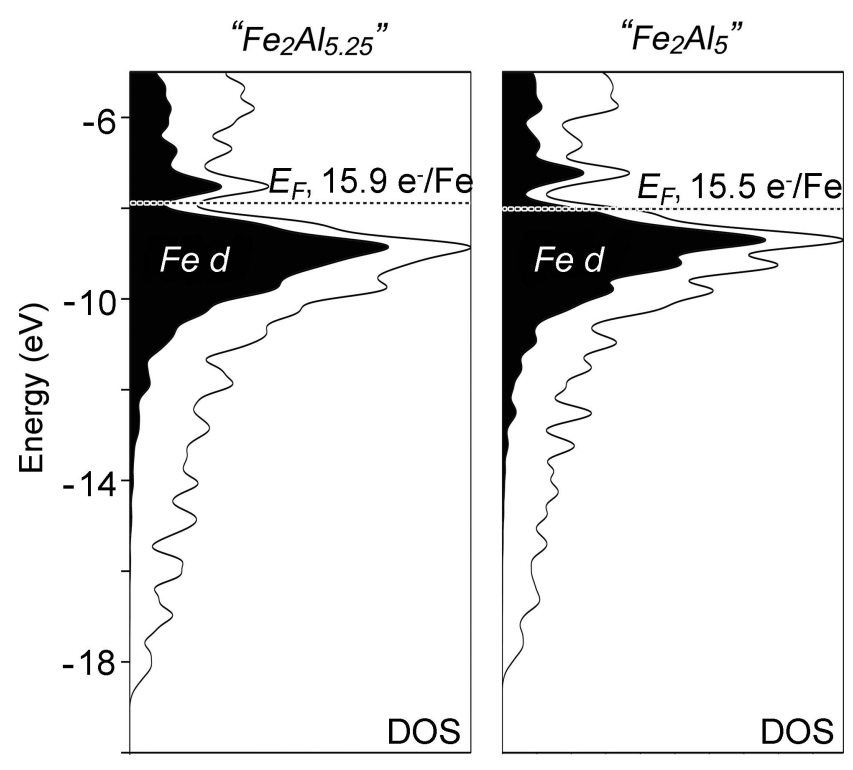

Figure 4

DFT electronic DOS distributions for two ordered models of $\mathrm{Fe}_{2} \mathrm{Al}_{5}$ (left: Model 1, right: Model 2). Gaussian broadening has been applied here (and in other DOS curves in this work) to bring out their more general features. 

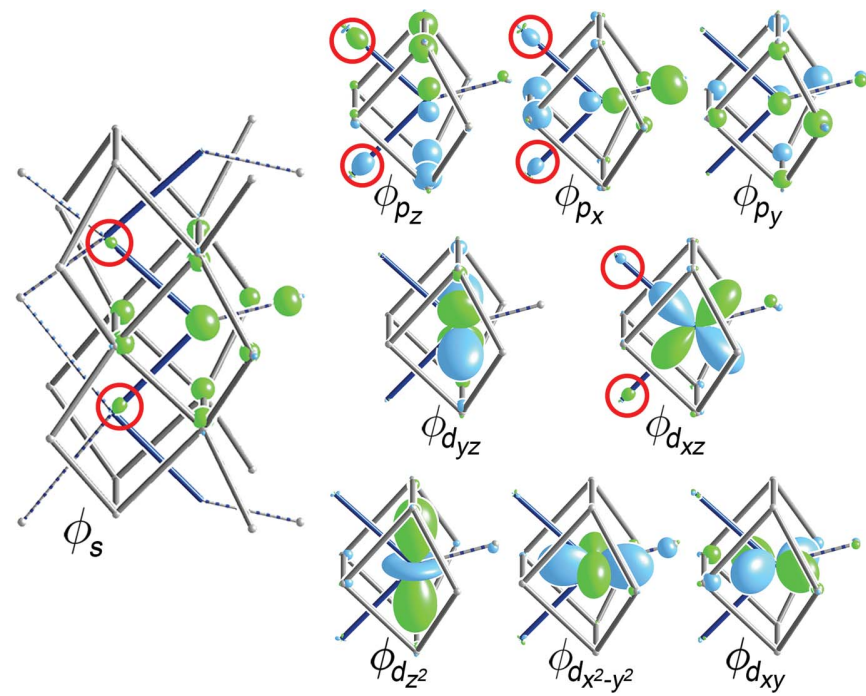

Figure 5

raMO reconstructions of the $4 s, 4 p$ and $3 d$ valence orbitals for an Fe atom in the $\mathrm{Fe}_{2} \mathrm{Al}_{5}$ phase (Model 1, composition: $\mathrm{Fe}_{2} \mathrm{Al}_{5.25}$ ). Substantial contributions from neighboring $\mathrm{Fe}$ sites are circled in red. Additional raMO results are provided in the supporting information.

original targets reflecting their bonding context and occupation.

In Fig. 5, we show the raMO reconstructions of the nine valence $s, p$ and $d$ orbitals for an Fe atom in Model 1, generated using a DFT-calibrated Hückel model. At the center of each raMO, the original target atomic orbital can be seen serving as the core of a function that spreads to varying degrees around the first coordination environment through bonding interactions. This localization of the function to the $\mathrm{Fe}$ atom and its immediate surroundings is consistent with the notion that each of these states is occupied by an electron pair, providing the Fe atom with a filled 18-electron configuration.

However, this configuration cannot be completely assigned to the central $\mathrm{Fe}$ atom independently: four of the raMOs (those based on the $4 s, 4 p_{x}, 4 p_{z}$ and $3 d_{x z}$ orbitals) exhibit bonding contributions from the neighboring $\mathrm{Fe}$ atoms in the zigzag chain, indicative of electron sharing between the $\mathrm{Fe}$ atoms (Fig. 5, red circles). The four atomic orbitals at the centers of these raMOs in fact form a set appropriate to the creation of $s p^{2} d$ hybrid orbitals pointing to the corners of a square, which would closely match the $\sim 90^{\circ} \mathrm{Fe}-\mathrm{Fe}-\mathrm{Fe}$ angles in the chains. Taking such linear combinations of these four raMOs produces two bonding functions localized along individual $\mathrm{Fe}-\mathrm{Fe}$ contacts representing $\mathrm{Fe}-\mathrm{Fe}$ isolobal $\sigma$ bonds (Fig. 6, top). The remaining two states generated by these linear combinations have the lobes pointing outwards from the $\mathrm{Fe}$ chain towards the neighboring Al2 column (Fig. 6, bottom). These would be considered non-bonding $\mathrm{Fe}-\mathrm{Fe}$ interactions, resembling $\mathrm{Fe}$ lone pairs that coordinate to the surrounding $\mathrm{Al}$ atoms. The remaining five raMOs not involved in this hybridization are also classified as $\mathrm{Fe}-\mathrm{Fe}$ nonbonding functions.

The two Fe-Fe isolobal bonds emerging from this raMO analysis concur with the hypothesis that $\mathrm{Fe}_{2} \mathrm{Al}_{5}$ follows the

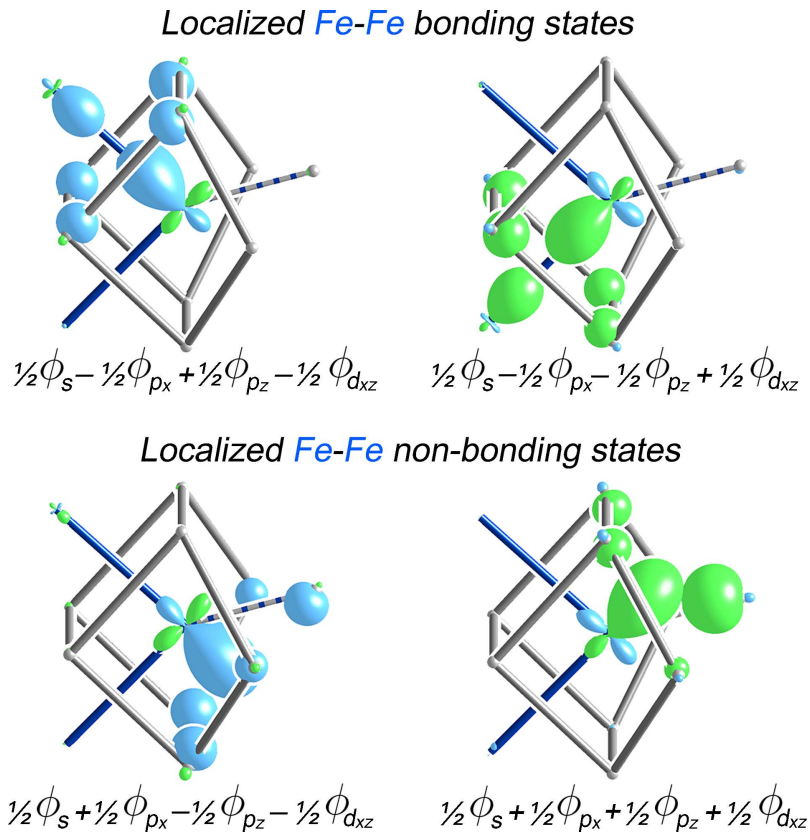

Figure 6

$s p^{2} d$ hybrids constructed from the raMOs in Fig. 5, revealing two isolobal $\mathrm{Fe}-\mathrm{Fe}$ bonds, as well as two $\mathrm{Fe}-\mathrm{Fe}$ nonbonding functions.

$18-n$ bonding scheme with $n=2$, such that the electronic structure is optimized at 16 electrons/Fe atom. As such, the reasons for the nonstoichiometry in the $\mathrm{Al}$ sublattice become apparent. A perfect 16 electron count requires a stoichiometry of $\mathrm{Fe}_{2} \mathrm{Al}_{5.33}$, which corresponds to a desired $\mathrm{Al}$ content of 10.667 atoms (a non-integer number) for the four $\mathrm{Fe}$ atoms within the $\mathrm{Fe}_{2} \mathrm{Al}_{5}$ unit cell. The Al-rich side of the experimentally observed homogeneity range, with electron concentrations up to 16.1, coincides closely with filling the band structure up to the top of the pseudogap. Adding more $\mathrm{Al}$ atoms into the system would then be expected to populate $\mathrm{Fe}-$ Fe $\sigma^{*}$ antibonding states, destabilizing the structure. The phase appears to be more flexible with lower electron counts, with electron counts as low as 15.0 in Al-poor compositions apparently being accessible. This greater flexibility towards electron-poor configurations over electron-rich ones is commonly observed in structures governed by the $18-n$ bonding scheme (Yannello \& Fredrickson, 2015), as the lower electron counts can open opportunities for isolobal $\pi$ interactions.

\subsection{Chemical pressure quadrupoles along the disordered channel}

Thus far, we have traced the $\mathrm{Al}$ nonstoichiometry in $\mathrm{Fe}_{2} \mathrm{Al}_{5}$ to the phase's adherence to the $18-n$ bonding scheme. This picture, however, focuses almost entirely on the Fe atoms and their placement relative to each other; the Al atoms merely serve to provide electrons and support functions whose symmetry properties are templated by the Fe atomic orbitals. As such, we gain little information about how the Al nonstoichiometry should be distributed through the unit cell and, in particular, why it becomes concentrated into weakly 
ordered columns running through the structure. In this section, we will see how the DFT-CP analysis accounts for these observations and suggests a general framework for identifying portions of a structure susceptible to weak positional order.

In the $\mathrm{CP}$ analysis, the local strains in the atomic packing of a compound are visualized by decomposing its macroscopic pressure, as obtained from DFT, into a spatially resolved map of regions of negative and positive pressure calling for contraction and expansion of the structure, respectively. These local pressures are analyzed in terms of interatomic interactions by dividing space into volumes corresponding to pairs of atoms and averaging the pressures within to obtain interatomic pressures, which are then projected onto spherical harmonics. The pressures experienced by each atom are finally displayed with radial plots, where black lobes represent negative CP (desire for contraction), white ones denote positive CP (desire for expansion), and the distance from the nucleus to the surface is proportional to the sum of the $\mathrm{CP}$ contributions along each direction.

In Fig. 7, we show as an example the CP scheme for one model of $\mathrm{Fe}_{2} \mathrm{Al}_{5}$ in which $\mathrm{Al}$ atoms are placed on one half of the Al2a sites (Model 2). The $\mathrm{Fe}-\mathrm{Al}$ interactions are marked by prominent lobes of positive $\mathrm{CP}$ calling for expansion of the structure - strongest between $\mathrm{Fe}$ and the Al2a atoms in the channel but also present between $\mathrm{Fe}$ and the $\mathrm{Al}$ atoms in the $\mathrm{Fe}_{2} \mathrm{Al}_{4}$ host lattice. These calls, however, are resisted by negative pressures on the $\mathrm{Al}$ atoms that, while less focused, lie along $\mathrm{Al}-\mathrm{Al}$ interactions. The major tension in the atomic packing of $\mathrm{Fe}_{2} \mathrm{Al}_{5}$ thus seems to stem from the overly compressed $\mathrm{Fe}-\mathrm{Al}$ interactions whose release is stymied by the already too-stretched $\mathrm{Al}$ sublattice.

One of the most striking aspects of this $\mathrm{CP}$ scheme is the distribution of pressures around the Al2a sites. These atoms are placed directly between two $\mathrm{Fe}$ neighbors on opposite sides of the channel. This results in the $\mathrm{Al}$ atom experiencing strong positive CPs along its close $\mathrm{Al}-\mathrm{Fe}$ contacts, pinning it to the center of the channel. Meanwhile, negative $\mathrm{CP}$ features on the $\mathrm{Al}$ atoms are perpendicular to the positive $\mathrm{CP}$ and slanted along the local direction of the channel, reflecting the sparseness of the $\mathrm{Al}$ channel population. Such a quadrupolar CP distribution is frequently associated with soft atomic motions, as shifts along the direction of negative pressure would serve to shorten some overly long contacts while relieving positive CPs in the perpendicular direction.

To explore this possibility of soft motions, we carried out

\section{Chemical Pressure Scheme of $\mathrm{Fe}_{2} \mathrm{Al}_{5}$ $1 / 2$ Al2a sites occupied}

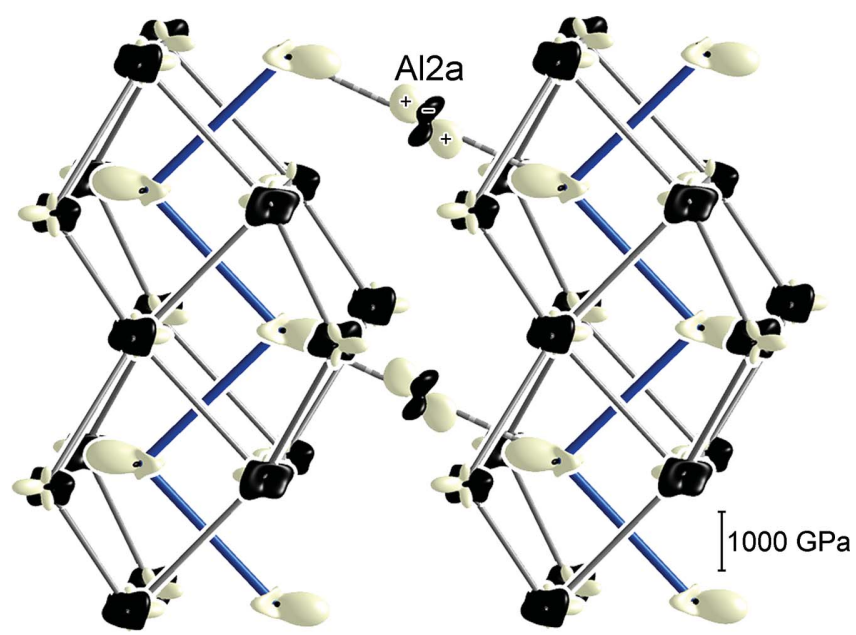

Figure 7

DFT-CP scheme for an ordered model of $\mathrm{Fe}_{2} \mathrm{Al}_{5}$ in which $\mathrm{Al}$ atoms are placed at one half of the Al2a sites (Model 2, see Fig. 1). The conventions of the $\mathrm{CP}$ plots are described in the text.

calculations on the phonon band structure of this ordered model of $\mathrm{Fe}_{2} \mathrm{Al}_{5}$ (Fig. 8a). As $\mathrm{Al}$ is significantly lighter than $\mathrm{Fe}$, we would normally expect Al-based motions to dominate the high-frequency modes, with the $\mathrm{Fe}$ atoms participating in the low-frequency vibrations. It is remarkable, then, that the lowest peak in the DOS stems chiefly from the motions of the Al2 sites (shaded in gray) in a single low-frequency band.

To examine the character of this band, we inspect its phonon mode at the $q$-point $T\left(\frac{1}{2} \frac{1}{2} \frac{1}{2}\right)$ in the top panel of Fig. 8(b), where the atomic motions are represented with arrows. The largest amplitudes appear on the $\mathrm{Al} 2$ atoms along directions of negative $\mathrm{CP}$, corresponding to a motion along the

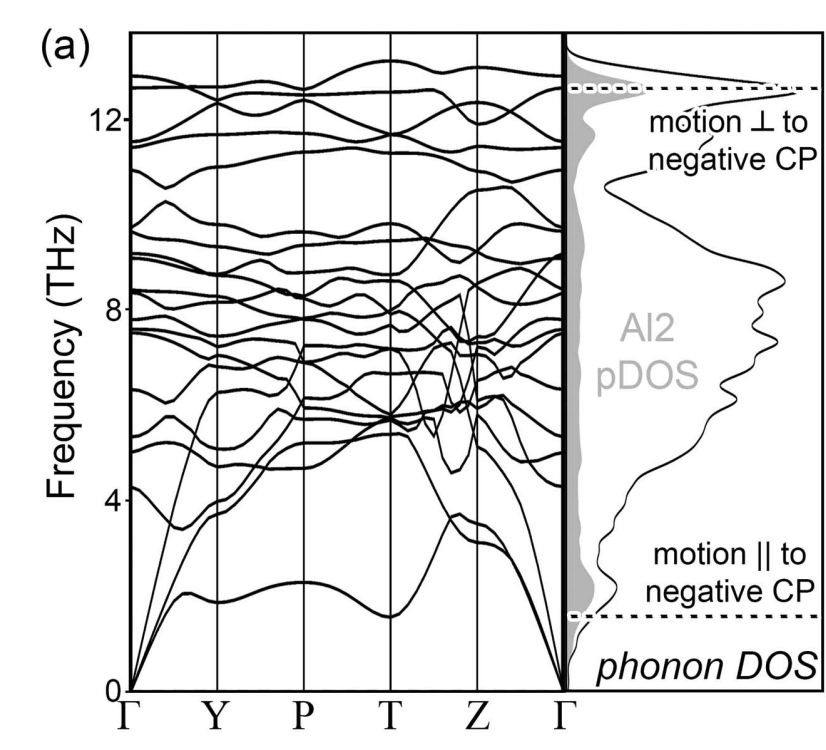

Figure 8

Connection between CP quadrupoles and phonon modes. (a) Phonon band structure and DOS for the model $\mathrm{Fe}_{2} \mathrm{Al}_{5}$ compound with one half of the Al2a sites occupied (Model 2). The projected phonon DOS for the Al2a site is highlighted in gray. (b) Selected phonon modes from the peaks in the Al2a projected DOS show anisotropy that aligns with the CP quadrupole of the Al2a site (overlaid). 
undulating Al2 disordered column in the experimental structure. A second peak with substantial Al2 contributions can be found in the phonon DOS near the top of the band structure at circa $12.7 \mathrm{THz}$. In stark contrast to the character of the lowfrequency mode, these modes correspond to motions of the Al2 atom directly along the positive $\mathrm{Fe}-\mathrm{Al} \mathrm{CP}$ [Fig. 8(b), bottom]. The anisotropy of the $\mathrm{CP}$ distribution around the $\mathrm{Al} 2$ atoms, with negative $\mathrm{CP}$ along the channel and positive $\mathrm{CP}$ towards the Fe-lined channel walls, is therefore mirrored in the site's freedom of motion. Motions along the direction of positive $\mathrm{CP}$ are stiff and high frequency, while those along the direction of negative $\mathrm{CP}$ are much more fluid.

In Fig. 9, we shift our attention to the CP features for this region of the structure with other $\mathrm{Al} 2$ channel occupation patterns. In our next model [Fig. 9(b), Model 3], $\mathrm{Al}$ atoms are placed on every other Al2b site. One large positive CP is present on each of these atoms, oriented towards the nearest

\section{CP schemes of $\mathrm{Fe}_{2} \mathrm{Al}_{4}+\delta$ models}

(a) $\delta=1,1 / 2$ Al2a sites occupied
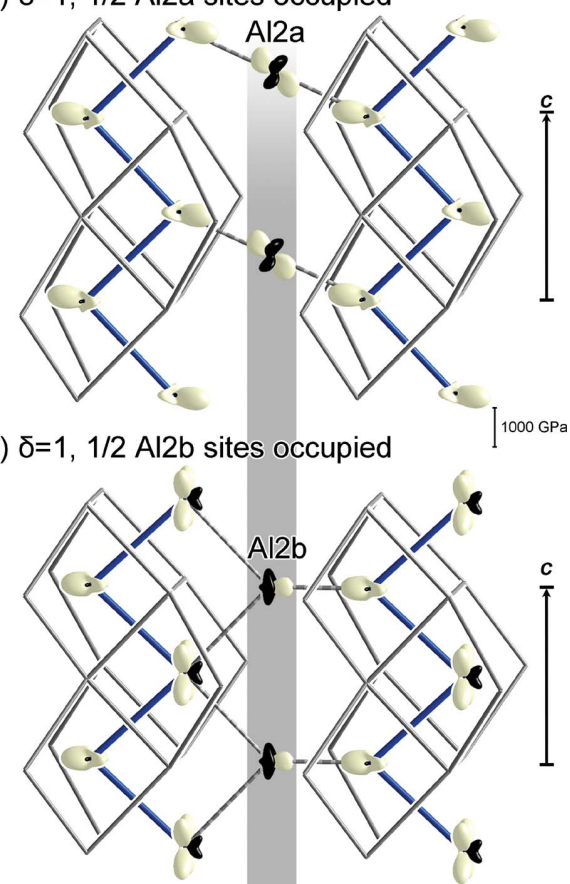

(c) $\delta=1.25,5 / 16$ Al2c sites occupied

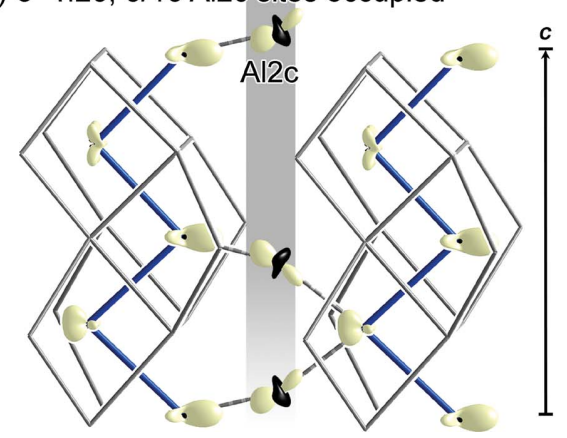

Figure 9

DFT-CPs underlying channel formation in $\mathrm{Fe}_{2} \mathrm{Al}_{5}$. The CPs experienced by the $\mathrm{Fe}$ and $\mathrm{Al} 2$ atom sites are shown in three ordered models: $(a)$ Model 2, (b) Model 3 and (c) Model 1. A persistent feature is the presence of negative $\mathrm{CP}$ along the $\mathrm{Al} 2$ channel.
Fe neighbor. Although the Al2b site has shifted from the center of the channel (where the Al2a atoms are held) in response to this $\mathrm{Fe}$ atom, the growth of negative $\mathrm{Al}-\mathrm{Al} \mathrm{CPs}$ appears to prevent full relief of the $\mathrm{Fe}-\mathrm{Al}$ positive $\mathrm{CP}$. As in the previous model (Fig. 9a), negative CPs are also directed perpendicular to the $\mathrm{Fe}-\mathrm{Al}$ contacts, with a substantial component along the direction of diffuse electron density in the experimental structure.

The role of the Al2c site in the structural solution as a bridge between the Al2a and Al2b sites is reflected by its $\mathrm{CP}$ scheme in the more complicated superstructure of Model 1 (Fig. 9c), where 5/16 of the Al2c sites are occupied. Yet again, strong positive CPs are directed towards the nearest $\mathrm{Fe}$ neighbors, while negative CPs are directed along the local path of the channel, and the distribution appears as an intermediate between the Al2a and Al2b CP schemes.

In all three models, then, the placement of perpendicular positive and negative $\mathrm{CP}$ lobes on the $\mathrm{Al} 2$ atoms produces a feature strongly correlated with soft phonon modes, a CP quadrupole. Intriguingly, these motions would tend to guide the Al2a site into the Al2b site (via the Al2c site) and vice versa. Such a path is emphasized in Fig. 10, where Al2 CPs from these three models are plotted within an isosurface of the Fourier electron density for this region from the experimental diffraction data. The negative pressure features of the $\mathrm{CP}$ distributions align closely with the continuous path of the electron density, hinting at easy diffusion of $\mathrm{Al}$ atoms along the channel between the Fe zigzag chains. This scheme also provides a rationale for the facile motions of $\mathrm{Al}$ atoms along these channels observed in earlier phonon calculations (Mihalkovič \& Widom, 2012).

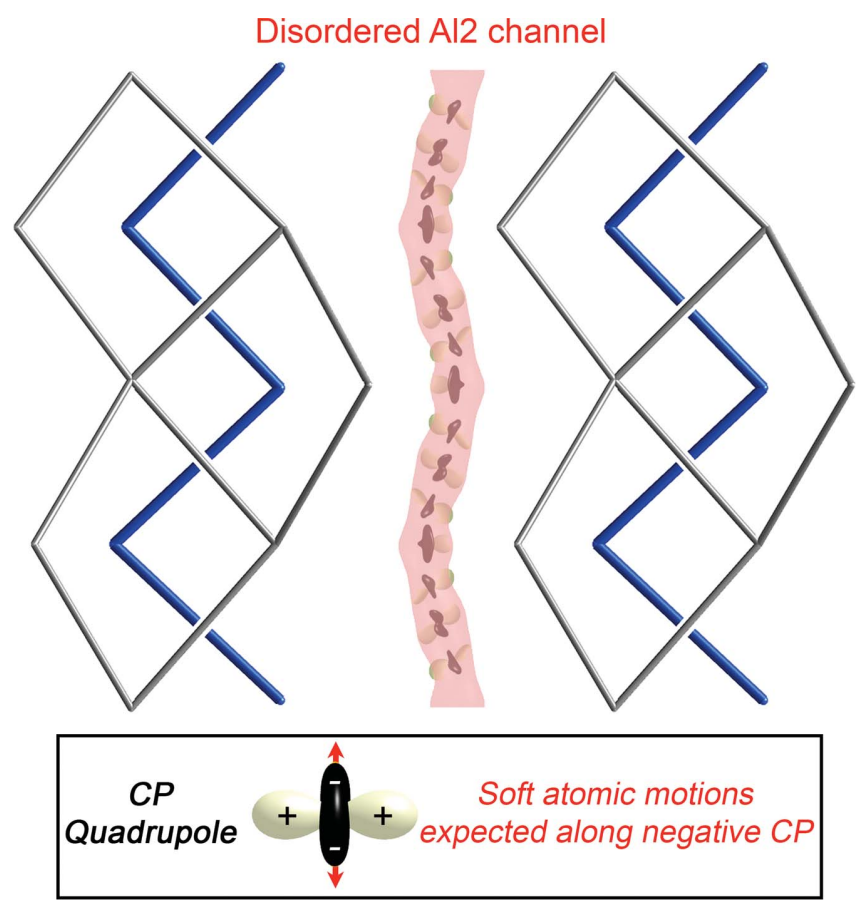

Figure 10

Placement of the $\mathrm{A} 12 \mathrm{CP}$ distributions from the various ordered models within the experimental $\mathrm{Fe}_{2} \mathrm{Al}_{5}$ structure. A Fourier electron-density isosurface for the disordered region is shown for $\rho=5.0$ e $\AA^{-3}$. 
Overall, the CP distributions of these ordered models of $\mathrm{Fe}_{2} \mathrm{Al}_{5}$ reveal $\mathrm{CP}$ quadrupoles on the $\mathrm{Al} 2$ sites which describe an undulating path along $c$. When we recall that optimizing the bonding scheme of this compound requires nonstoichiometry on the Al sublattice, this region of the structure appears ideally suited to accommodate it. The lack of a clear preference for any particular positioning of the $\mathrm{Al}$ atoms within the channel, except to maximize $\mathrm{Fe}-\mathrm{Al}$ distances, anticipates the weak positional order along it. The ease with which atoms move along the path also means that they would have little trouble shifting in response to the addition or removal of another $\mathrm{Al}$ atom in the channel, accounting for the observed compositional range of the phase.

\section{Conclusions}

This work was inspired by the curious disordered channels of $\mathrm{Al}$ atoms that appear in the high-temperature structure of $\mathrm{Fe}_{2} \mathrm{Al}_{5}$ and contribute to its promise as a thermoelectric material. After confirming an experimental model in which the $\mathrm{Al}$ atoms are disordered in an undulating pattern throughout the compound, we applied a series of theoretical methods to elucidate the role of this channel in stabilizing the structure. Using the raMO analysis, we traced the nonstoichiometry of the $\mathrm{Al}$ content to the desire by the $\mathrm{Fe}$ bonding network to achieve 18-electron configurations, following the $18-n$ rule. DFT-CP schemes then revealed how the channels in the structure accommodate the Al2 disorder: the placement of these sites between zigzag chains of $\mathrm{Fe}$ atoms leads to $\mathrm{CP}$ quadrupoles that allow for soft motions threading $\mathrm{Al}$ atoms along the path defined by the Fe.

The way that electron counts and chemical pressures conspire to produce the disordered Al2 columns suggests an approach by which structures with similar order/disorder dichotomies could be induced in a wide range of intermetallic phases. Compounds that follow the $18-n$ bonding scheme while exhibiting clusters, columns, networks or sheets of CP quadrupoles on some of the main group sites would be expected to be responsive to elemental substitutions affecting the electron concentration. In such cases, motions within the soft sublattices defined by the CP quadrupoles would provide a path by which main group atoms could be inserted or removed to maintain the $18-n$ electron counts (with their sizes also of potential importance). We are looking forward to exploring how this idea might apply to such structural series as the Nowotny Chimney Ladder phases (Nowotny, 1970), and to the discovery of new intermetallic compounds.

\section{Related literature}

The following references are cited in the supporting information: Bruker (2016), Rigaku (2015), von Goldbeck (1982), Grin et al. (1994), Petříček et al. (2014), Sheldrick (1996, 2015a,b).

\section{Acknowledgements}

We thank Dr John Fournelle for assistance with the WDS measurements. We also thank Dr Vincent Yannello for insightful discussions of the application of the raMO method, and Erdong $\mathrm{Lu}$ for conversations regarding the interaction between the $18-n$ bonding scheme and $\mathrm{CP}$ quadrupoles in shaping intermetallic structures.

\section{Funding information}

We gratefully acknowledge the financial support of the National Science Foundation through grant DMR-1508496. This research involved calculations using computer resources supported by National Science Foundation Grant CHE0840494.

\section{References}

Bader, R. F. W. (1990). Atoms in Molecules: A Quantum Theory. Oxford University Press.

Becker, H., Amirkhanyan, L., Kortus, J. \& Leineweber, A. (2017). J. Alloys Compd. 721, 691-696.

Becker, H. \& Leineweber, A. (2018). Intermetallics, 93, 251-262.

Berns, V. M., Engelkemier, J., Guo, Y., Kilduff, B. J. \& Fredrickson, D. C. (2014). J. Chem. Theory Comput. 10, 3380-3392.

Blöchl, P. E. (1994). Phys. Rev. B, 50, 17953-17979.

Bruker (2016). APEX3. Bruker AXS, Madison, Wisconsin, USA.

Burkhardt, U., Grin, Yu., Ellner, M. \& Peters, K. (1994). Acta Cryst. B50, 313-316.

Chi, J., Zheng, X., Rodriguez, S. Y., Li, Y., Gou, W., Goruganti, V., Rathnayaka, K. D. D. \& Ross, J. H. (2010). Phys. Rev. B, 82, 174419.

Ellner, M. \& Mayer, J. (1992). Scr. Metall. Mater. 26, 501-504.

Engelkemier, J. \& Fredrickson, D. C. (2016). Chem. Mater. 28, 31713183.

Feuerbacher, M., Thomas, C., Makongo, J. P. A., Hoffmann, S., Carrillo-Cabrera, W., Cardoso, R., Grin, Y., Kreiner, G., Joubert, J. M., Schenk, T., Gastaldi, J., Nguyen-Thi, H., Mangelinck-Noel, N., Billia, B., Donnadieu, P., Czyrska-Filemonowicz, A., ZielinskaLipiec, A., Dubiel, B., Weber, T., Schaub, P., Krauss, G., Gramlich, V., Christensen, J., Lidin, S., Fredrickson, D., Mihalkovic, M., Sikora, W., Malinowski, J., Bruhne, S., Proffen, T., Assmus, W., de Boissieu, M., Bley, F., Chemin, J. L., Schreuer, J. \& Steurer, W. (2007). Z. Kristallogr. 222, 259-288.

Fredrickson, D. C. (2012). J. Am. Chem. Soc. 134, 5991-5999.

Giannozzi, P., de Gironcoli, S., Pavone, P. \& Baroni, S. (1991). Phys. Rev. B, 43, 7231-7242.

Goldbeck, O. K. von (1982). Iron-Binary Phase Diagrams. Aachen: Springer-Verlag.

Gómez, C. P. \& Lidin, S. (2003). Phys. Rev. B, 68, 024203.

Gonze, X., Amadon, B., Anglade, P. M., Beuken, J. M., Bottin, F., Boulanger, P., Bruneval, F., Caliste, D., Caracas, R., Côté, M., Deutsch, T., Genovese, L., Ghosez, P., Giantomassi, M., Goedecker, S., Hamann, D. R., Hermet, P., Jollet, F., Jomard, G., Leroux, S., Mancini, M., Mazevet, S., Oliveira, M. J. T., Onida, G., Pouillon, Y., Rangel, T., Rignanese, G. M., Sangalli, D., Shaltaf, R., Torrent, M., Verstraete, M. J., Zerah, G. \& Zwanziger, J. W. (2009). Comput. Phys. Commun. 180, 2582-2615.

Gonze, X., Beuken, J. M., Caracas, R., Detraux, F., Fuchs, M., Rignanese, G. M., Sindic, L., Verstraete, M., Zerah, G., Jollet, F., Torrent, M., Roy, A., Mikami, M., Ghosez, P., Raty, J. Y. \& Allan, D. C. (2002). Comput. Mater. Sci. 25, 478-492.

Gray, D. L., Francisco, M. C. \& Kanatzidis, M. G. (2008). Inorg. Chem. 47, 7243-7248. 
Grin, J., Burkhardt, U., Ellner, M. \& Peters, K. (1994). Z. Kristallogr. Cryst. Mater. 209, 479.

Hartwigsen, C., Goedecker, S. \& Hutter, J. (1998). Phys. Rev. B, 58, 3641-3662.

Henkelman, G., Arnaldsson, A. \& Jónsson, H. (2006). Comput. Mater. Sci. 36, 254-360.

Hilleke, K. P. \& Fredrickson, D. C. (2018). J. Phys. Chem. A, 122, 8412-8426.

Hirshfeld, F. L. (1977). Theor. Chim. Acta, 44, 129-138.

Jagličić, Z., Vrtnik, S., Feuerbacher, M. \& Dolinšek, J. (2011). Phys. Rev. B, 83, 224427.

Kanno, M., Yamada, T., Ikeda, T., Nagai, H. \& Yamane, H. (2017). Chem. Mater. 29, 859-866.

Kilduff, B. J. \& Fredrickson, D. C. (2016). Inorg. Chem. 55, 67816793.

Kitahara, K., Takagiwa, Y. \& Kimura, K. (2015). J. Phys. Soc. Jpn, 84, 014703.

Kitahara, K., Takagiwa, Y. \& Kimura, K. (2017). J. Phys. Conf. Ser. 809, 012014.

Kresse, G. \& Furthmüller, J. (1996a). Comput. Mater. Sci. 6, 15-50.

Kresse, G. \& Furthmüller, J. (1996b). Phys. Rev. B, 54, 11169-11186.

Kresse, G. \& Joubert, D. (1999). Phys. Rev. B, 59, 1758-1775.

Latturner, S. E. \& Kanatzidis, M. G. (2002). Inorg. Chem. 41, 54795486.

Li, X., Scherf, A., Heilmaier, M. \& Stein, F. (2016). J. Phase Equilib. Diffus. 37, 162-173.

Mählpfordt, W. (1997). Z. Anorg. Allg. Chem. 623, 985-989.

Mehrer, H. (1996). Mater. Trans. JIM, 37, 1259-1280.

Mihalkovič, M. \& Widom, M. (2012). Phys. Rev. B, 85, 014113.

Nolas, G. S., Morelli, D. T. \& Tritt, T. M. (1999). Annu. Rev. Mater. Sci. 29, 89-116.

Nowotny, H. (1970). The Chemistry of Extended Defects in Nonmetallic Solids, edited by L. Eyring \& M. O'Keeffe. Amsterdam and London: North-Holland Publishing Co.

Okamoto, N. L., Okumura, J., Higashi, M. \& Inui, H. (2017). Acta Mater. 129, 290-299.
Oliveira, M. J. T. \& Nogueira, F. (2008). Comput. Phys. Commun. 178, 524-534.

Osawa, A. (1933). Sci. Rep. Tohoku Univ. 22, 803-823.

Petř́íček, V., Dušek, M. \& Palatinus, L. (2014). Z. Kristallogr. Cryst. Mater. 229, 345-352.

Piao, S. Y., Gómez, C. P. \& Lidin, S. (2006). Z. Naturforsch. Teil B, 61, 644-649.

Piao, S. Y. \& Lidin, S. (2007). Philos. Mag. 87, 2693-2699.

Rigaku (2015). CrysAlis Pro. Version 171.37.35. Rigaku Oxford Diffraction, Yarnton, UK.

Rohrer, F. E., Lind, H., Eriksson, L., Larsson, A. K. \& Lidin, S. (2001). Z. Kristallogr. Cryst. Mater. 216, 190.

Samson, S. (1965). Acta Cryst. 19, 401-413.

Schubert, K., Rösler, U., Kluge, M., Anderko, K. \& Härle, L. (1953). Naturwissenschaften, 40, 437.

Sheldrick, G. M. (1996). SADABS. University of Göttingen, Germany.

Sheldrick, G. M. (2015a). Acta Cryst. C71, 3-8.

Sheldrick, G. M. (2015b). Acta Cryst. A71, 3-8.

Stacey, T. E. \& Fredrickson, D. C. (2012). Dalton Trans. 41, 78017813.

Takakura, H., Gómez, C. P., Yamamoto, A., De Boissieu, M. \& Tsai, A. P. (2007). Nat. Mater. 6, 58-63.

Tang, W., Sanville, E. \& Henkelman, G. (2009). J. Phys. Condens. Matter, 21, 084204.

Tobita, K., Sato, N., Kitahara, K., Takagiwa, Y. \& Kimura, K. (2016). Mater. Trans. 57, 1045-1049.

Vanderbilt, D. (1990). Phys. Rev. B, 41, 7892-7895.

Van der Kraan, A. M. \& Buschow, K. H. J. (1986). Physica B, 138, 55 62.

Yannello, V. J. \& Fredrickson, D. C. (2014). Inorg. Chem. 53, $10627-$ 10631.

Yannello, V. J. \& Fredrickson, D. C. (2015). Inorg. Chem. 54, 1138511398.

Yannello, V. J., Kilduff, B. J. \& Fredrickson, D. C. (2014). Inorg. Chem. 53, 2730-2741. 